\title{
Nonionic detergent micelle aggregates: an economical alternative to Protein A chromatography
}

\author{
Guy Patchornik ${ }^{1}$, Gunasekaran Dhandapani ${ }^{1}$, Ellen Wachtel ${ }^{2}$, and Mordechai Sheves $^{3}$ \\ ${ }^{1}$ Ariel University Faculty of Natural Sciences \\ ${ }^{2}$ Weizmann Institute of Science Department of Biological Chemistry \\ ${ }^{3}$ Weizmann Institute of Science Faculty of Chemistry
}

May 5, 2020

\begin{abstract}
We have recently described a non-chromatographic, ligand-free approach for antibody (Ab) purification based on specially designed: [Tween-20:bathophenanthroline:Fe2+] aggregates. To assess the potential generality of this approach, a variety of detergents belonging to four nonionic detergent families (Tween, Brij, Triton and Pluronic) have now been studied. All surfactant aggregates lead to high purity of the recovered Abs ( $>95 \%$, by gel densitometry). Good overall Ab recovery yields were observed with: Tween-20 (80-83\%), Brij-O20 (85-87\%) and Triton X-100 (87-90\%), while Pluronic F-127 was less efficient (42-53\%). Of additional importance is the finding that the process can depend on filtration (rather than centrifugation), thereby allowing a continuous purification mode that leads to the recovery of monomeric IgG's (by DLS) and preservation of Ab specificity (by ELISA). The amphiphilic chelator, bathophenanthroline (batho) is recycled almost quantitatively (95\%) by crystallization. Good IgG recovery yields $(\sim 80 \%)$ are also observed when Ab concentrations are increased from $1 \mathrm{mg} / \mathrm{ml}$ to $3-5 \mathrm{mg} / \mathrm{mL}$. Potential advantages of the purification platform for industrial downstream processing of therapeutic monoclonal antibodies (mAbs), are discussed.
\end{abstract}

\section{Keywords}

Antibody purification, IgG, Protein A, chromatography, engineered-micelles.

\section{Introduction}

Antibodies (Abs) are routinely used as detection and quantitation agents in scientific research and as vehicles for delivering radioisotopes, drugs and enzymes to cells of interest in medicine. ${ }^{1-2}$ It is the inherent high specificity combined with the strong binding affinity towards diverse antigens (proteins, carbohydrates, nucleotides and haptens) that explains why personalized medicine relies to a great extent on monoclonal antibodies (mAbs), ${ }^{3}$ many of which find their use in cancer therapy. ${ }^{4-5}$ The striking success of mAbs as efficient therapeutic agents resulted in $61 \mathrm{FDA}$ - approved mAbs by $2017^{6}$ and $~ 570 \mathrm{mAbs}$ are currently in advanced clinical trials. ${ }^{7}$ The increasing global demand for diverse mAbs pressures pharmaceutical companies to improve the expression levels of mAbs (upstream processing) and their purification (downstream processing).

Antibody purification is most commonly achieved viachromatography using Protein A (ProA) as the ligand of choice for capturing Abs. ProA binds strongly ${ }^{8}$ and specifically ${ }^{9}$ to diverse Ab subclasses, ${ }^{10}$ making it possible to efficiently capture Abs from very complex media and to reach high purity $(>98 \%)$ within a single chromatographic step. ${ }^{11}$ These features have thus made ProA chromatography the gold standard technology in Ab purification. ${ }^{12}$ However, the necessity to purify ton amounts ${ }^{13}$ of therapeutic mAbs has led pharmaceutical companies to search for a more economical route to meet the above challenge. The ideal 
purification technology would thus rely on a non-chromatographic step capable of efficiently capturing Abs. This challenging objective was termed the: "ABC philosophy",i.e., Anything But Chromatography. ${ }^{12,}{ }^{14} \mathrm{We}$ sought to take this philosophy an additional step forward by entirely excluding ProA from the process.

Development of a non-chromatographic, ligand-free Ab purification platform has the potential of overcoming major problems related to ProA chromatography. These include: (i) the high cost of ProA resins and the potential leaching of ProA (or its fragments) into the purified $\mathrm{Ab} ;{ }^{12}$ (ii) the limited binding capacities of ProA columns which may pose a problem at high Ab concentrations; ${ }^{15}$ (iii) deamidation of ProA asparagines during column sanitation, ${ }^{16}$ leading to lower binding efficiency; and (iv) the high maintenance costs associated with the use of HPLC $\backslash$ FPLC instrumentation.

Great efforts have been directed toward replacing ProA chromatography with a non-chromatographic approach. These included: (a) diverse precipitation methods; ${ }^{17}$ (b) extraction systems; ${ }^{18-19}$ and (c) ultrafiltration with charged membranes; ${ }^{20}$ but none of these are widely used.

Recently, we described an alternative $\mathrm{Ab}$ purification platform that complies with such challenging criteria. ${ }^{21-22}$ Our approach relied on literature reports describing purification of $\mathrm{mAbs}$ viahydrophobic interaction chromatography (HIC) ${ }^{23-25}$ or hydrophobic membrane interaction chromatography. ${ }^{26}$ Those studies demonstrated the tendency of immunoglobulins (IgGs) to bind more strongly to non-polar surfaces than do non-IgG water-soluble proteins. We exploited this greater binding affinity of IgGs to hydrophobic resins by demonstrating that Tween-20 detergent micellar aggregates can be used for purification of human, mouse, rabbit and sheep IgGs. ${ }^{21-22}$

Tween-20 aggregates were formed in two-steps: first, the amphiphilic chelator, bathophenanthroline (batho), was added to a dispersion of Tween-20 micelles and transformed the latter into what we have termed: 'engineered-micelles', ${ }^{27-29}$ in which the chelator is presumably positioned at the micelle/water interface. This was followed by specific conjugation of engineered-micelles with $\mathrm{Fe}^{2+}$ ions (known to bind with high affinity up to three batho molecules in paralle ${ }^{14,30}$ ) leading to Tween-20 aggregates as a distinct amphiphilic phase. The latter were shown to efficiently capture IgG's from hybridoma serum-free media, or from E. coli lysate, and allow extraction of pure target IgG's without concomitant extraction of impurities or aggregate dissolution. ${ }^{21-22}$

In the present report, we demonstrate the generality of our non-chromatographic, ligand-free approach, as well as providing evidence which would further justify efforts at up-scaling. Achieving these challenging objectives is expected to form the basis for considering integration of our purification platform into standard downstream processing of therapeutic grade mAbs on an industrial scale.

\section{Results and Discussion}

Surfactant structure influences recovery yield of $I g G$. In an attempt to reveal any significant relationship between IgG purity/yield and surfactant structure, we studied several different non-ionic surfactant families (Figure 1, B) i.e., Tween, Brij, Triton and Pluronic (triblock-copolymer) detergents. While a hydrophobic anchor in the form of a hydrocarbon chain of varying length is present in Tween, Brij and Triton surfactants it is not present in the same form in Pluronic F-127 triblock copolymer surfactants. Rather the polypropylene central block forms the micellar core, while the polyethylene oxide blocks form the hydrophilic shell (Figure 1, B). The number of ethylene glycol moieties per detergent monomer did not affect the purity of recovered IgG's (Figure 2, C-D lanes 2-10) but did influence overall yield. The most efficient detergents in each family, from the point of view of overall yield, were found to be: Tween-20 (hIgG- 75\%, mouse IgG- 74\%); Brij-O20 (hIgG-69\%, mouse IgG- 70\%); Brij-100 (hIgG-73\%, mouse IgG- 70\%) and Triton X-100 (hIgG-72\%, mouse IgG -69\%). Pluronic F-127 was the least efficient (hIgG -42\%, mouse IgG -53\%) (Figure 2, C-D). This trend was also observed when analyzing pellet composition immediately following the IgG capturing step, though the presence of PEG-6000 in the system, led to diffused migration of the heavy and light chains that made accurate quantification unreliable (Figure 2, A-B lanes 2-10). Here, Tween-20 with the shortest alkyl tail (12 carbons) produced higher overall yields in comparison to other Tween family members with longer alkyl tails (16-18 carbons) (Figure 2, C-D, lanes 2-5). Moreover, results from the Brij family clearly 
indicated that overall yields increase with increasing number of oxyethylene units. Accordingly, Brij-10 with $\sim 10$ oxyethylene units was less efficient than Brij-O20 or Brij-100 containing ${ }^{2} 20$ or $\sim 100$ oxyethylene units, respectively (Figure 2, C-D, lanes 6-8). It seems therefore that process efficiency is determined by several parameters that include both the length of the detergent hydrocarbon tail and the number of oxyethylene groups.

Influence of aggregate size. An additional parameter that may influence total yield is aggregate size. We recently reported that aggregates with size ranging between 100-200 nm do not efficiently capture IgG. ${ }^{22}$ It was therefore surprising to find that DLS characterized Pluronic F-127 aggregates as having the largest size $(1312 \mathrm{~nm})$ of all the detergents we studied: i.e., Tween $(355-685 \mathrm{~nm})$, Brij $(635-683 \mathrm{~nm})$, Triton X-100 (913 nm) (Figure 3, A-D) . Thus, the larger size of Pluronic F-127 aggregates apparently could not compensate for the block copolymer core/shell micelle structure.

Filtration. In order to be considered for industrial-scale, continuous production flows of therapeutic mAbs ${ }^{12}$, IgG capturing and extraction from the aggregates would preferably be accomplishedvia filtration rather than centrifugation. We therefore replaced centrifugation steps with filtration at three critical stages in our IgG purification protocol involving Tween-20, Brij-O20 and Triton X-100 surfactants (Figure 4): (i) aggregate incubation with the IgG/BSA mixture in hybridoma serum free media; (ii) removing residual unbound IgG and/or BSA from the aggregates via $20 \mathrm{mM}$ cold $\mathrm{NaCl}$ wash; (iii) recovering IgG with extraction buffer: 50 $\mathrm{mM}$ Gly in $30 \mathrm{mM} \mathrm{NaCl}$ at pH 3.8 during 5 minutes at room temperature. The results clearly indicated that filtration can indeed replace centrifugation. The purity of recovered hIgG (Figure 4, A lanes 2-7) was not different from that obtained with centrifugation (Figure 2, C-D lanes 2, 7, 9) while the overall yields improved and reached values ranging between $80-90 \%$ after two consecutive extraction steps (Figure 4, A). Of the three detergents tested, Triton X-100 repeatedly exhibited superiority over Tween-20 and Brij-O20 (Figure 4, A). Thus, a relatively pure Ab preparation can be conveniently and efficiently obtained via filtration using detergent aggregates belonging to three surfactant families. This finding emphasizes the wide scope of working conditions that can be employed with our purification platform.

Since pharmaceutical use of therapeutic mAbs requires these to be monomeric, ${ }^{31}$ recovered hIgG obtained with the filtration protocol was analyzed by dynamic light scattering (DLS). The results indicated that purified $\mathrm{hIgG}$ is indeed monomeric, as no significant difference in particle size was observed when compared to the control, pure hIgG which had not been subjected to the surfactant aggregate protocol (Figure 4, B). Particle sizes were in agreement with our previous findings ${ }^{32}$ and ranged between: 10.4-11.0 $\mathrm{nm}$ regardless of the detergent used (i.e. Tween-20, Brij-O20, Triton X-100).

Ab specificity. Though IgG purity, overall recovery yields and monomeric state are factors of paramount importance in the development of new Ab purification methodologies, preservation of Ab specificity must also be validated. This question was answered for a specific Ab (an anti-BSA IgG) using the detergent aggregate platform and the filtration protocol. Accordingly, the recovered anti-BSA was subjected to an ELISA assay in order to assess its capability in binding target (BSA). Positive signals in the ELISA assay were observed with all three aggregates (Figure 4, C) and reflected recovery yields similar to those obtained with human or mouse IgG's using identical detergent aggregates (Figure 4, A). Preservation of IgG specificity is not surprising when considering the presence of at least [?]14 disulfide bonds in all IgG isotypes. ${ }^{33}$ The fact that Tween-20, Brij-O20, Brij-100 and Triton X-100 repeatedly demonstrated their utility with polyclonal human and mouse IgG's suggests that the IgG capturing step is independent of antibody amino acid sequence. This is an important finding since it implies that a single nonionic detergent may provide a purification platform for many different antibodies, regardless of their biological origins. Such a capability is ideal for industrial downstream processing of therapeutic grade mAbs.

Increasing IgG concentration. The fact that $\mathrm{mAb}$ production in $\mathrm{CHO}$ cells has improved significantly and currently can reach $\mathrm{Ab}$ titers greater than $1 \mathrm{mg} / \mathrm{mL}^{34}$ led us to test our filtration protocol at IgG concentrations up to $5 \mathrm{mg} / \mathrm{ml}$ (Figure 5). As before, we focused on the three detergents that led to the highest recovery yields via the centrifugation protocol. The results presented in Figure 5 indicate that process efficiency at 3 $\mathrm{mg} / \mathrm{mL}$ and $5 \mathrm{mg} / \mathrm{mL}$ decreases by $5-10 \%$ relative to that obtained at $1 \mathrm{mg} / \mathrm{mL}$. Clearly, working at higher 
IgG concentrations requires a larger number of aggregated micelles. Indeed, good overall yields ( $~ 80 \%)$, necessitated an increase by a factor of 1.6 and 2.3 (wt/vol) for $3 \mathrm{mg} / \mathrm{ml}$ and $5 \mathrm{mg} / \mathrm{ml} \mathrm{hIgG}$, respectively. These results are encouraging as they are obtained at current industrial IgG expression levels. ${ }^{34}$

Chelator recycling. Recycling of raw material represents an important environmental and economic concern for large-scale pharmaceutical production. We therefore studied the possibility of regenerating the most costly component in our platform, i.e. the chelator (batho). Chelator recycling via crystallization appears to be both economical and realistic due to the inherent physiochemical properties of batho. These include: water-insolubility; ${ }^{35}$ stability under both acidic ${ }^{36}$ and basic conditions, and at elevated temperatures; ${ }^{37}$ and the ability of batho to crystallize rapidly in aqueous media due to its relatively rigid and extended aromatic system which is capable of forming $\pi-\pi$ interactions and thereby, aromatic stacking between batho molecules.

Tween-20 was the surfactant of choice for this recycling experiment. Initial removal of Tween-20 from the aggregates was essential for optimal batho recycling, as the presence of any detergent would likely improve batho's water-solubility and hence, suppress its crystallization in aqueous media. However, Tween-20 was found to be soluble even in $1 \mathrm{M} \mathrm{NaCl}$ (data not shown). Therefore, Tween-20 aggregates that were used for IgG capture and extraction were thoroughly washed with $0.25 \mathrm{M} \mathrm{NaCl}$ to remove the detergent as well as the IgG's that might not have been extracted from the aggregates. Under these conditions, the extremely hydrophobic [(batho $)_{3}: \mathrm{Fe}^{2+}$ ] red complex is obviously water-insoluble (Figure 5, Step I). The pellet size decreased after $\mathrm{NaCl}$ washing and the resulting, excluded aqueous solution was colorless, indicating no significant loss of the $\left[(\text { batho })_{3}: \mathrm{Fe}^{2+}\right]$ red complex (data not shown). Light microscopy analysis of washed aggregates showed that these are composed of numerous red crystals $\left({ }^{\sim} 30-70 \mu \mathrm{m}\right)$ (Figure $\left.7, \mathrm{~A}\right)$ containing the $\left[(\text { batho })_{3}: \mathrm{Fe}^{2+}\right]$ complex as determined by mass spectrometry (MS) (Supplementary S1). In order to dissociate the red complex, crystals were briefly $\left(2-3 \mathrm{~min}\right.$.) heated at $95{ }^{\circ} \mathrm{C}$ in $\mathrm{DDW} / \mathrm{MeOH}(1: 1 \mathrm{vol} / \mathrm{vol})$ containing $10 \mathrm{mM}$ EDTA. Upon cooling, the red color was no longer observed, being replaced by a colorless, transparent solution (not shown). Thus, the loss of the red color served as a convenient internal indicator for monitoring the rate and efficiency at which the $\left[(\text { batho })_{3}: \mathrm{Fe}^{2+}\right]$ red complex disintegrates in the presence of the competing EDTA chelator. It should be emphasized that the presence of $50 \% \mathrm{MeOH}$ in the aqueous medium is essential as it led to the complete dissolution of the red crystals and thus, physical access of EDTA to the chelated iron ions in the $\left[(\text { batho })_{3}: \mathrm{Fe}^{2+}\right]$ red complex. In order to then completely remove $\mathrm{MeOH}$, which suppresses batho crystallization, the solution was heated to $95{ }^{\circ} \mathrm{C}$ for 30 minutes and the resulting precipitate was thoroughly washed with DDW to remove EDTA, the [EDTA- $\mathrm{Fe}^{2+}$ ] colorless complex and free $\mathrm{Fe}^{2+}$ ions if present (Figure 5, Step IV). Analysis of the precipitate by light microscopy showed that it contains colorless, plate-like crystals (Figure 7, B) while liquid chromatography mass-spectrometry (LCMS) analysis confirmed that the crystals are indeed $>98 \%$ pure batho (Supplementary S2, C) identical to , or perhaps even purer than commercial batho crystals prior to use (Supplementary S2, D) (Figure 7, C). The fact that the MS spectrum of recycled batho (Supplementary S2, C) was essentially identical to that of the pure, commercial batho (Supplementary S2, A) implies that, no chemical modifications occurred during chelator regeneration. To further rule out possible chemical modifications of the chelator during the recycling procedure, regenerated batho crystals were dissolved in $\mathrm{MeOH}$ and $\mathrm{Fe}^{2+}$ ions were added to generate the $\left[(\text { batho })_{3}: \mathrm{Fe}^{2+}\right]$ red complex. The characteristic absorbance of the latter $\left(\lambda_{\max }=530 \mathrm{~nm}\right)$ was compared to the absorption spectrum of a freshly prepared $\left[(\text { batho })_{3}: \mathrm{Fe}^{2+}\right]$ red complex generated from the commercial (unused) chelator and no marked differences were observed (not shown). This finding provided additional supporting evidence for the preservation of the chelating ability of recycled crystalline batho. The characteristic absorption of the complex at $530 \mathrm{~nm}$ served as a means of quantitating chelator regeneration efficiency which was found to be, on average, $95 \%$ when tested in10 independent experiments (not shown). It should be emphasized that this value represents the recycling yield relative to the total amount of batho present initially in the system. These findings agree with the physiochemical properties of batho described above and show that near quantitative recycling can be achieved.

\section{Conclusions}

Detergent aggregates composed of conjugated Tween-20, Brij-O20 or Triton X-100 nonionic micelles and 
the amphiphilic [(batho $)_{3}: \mathrm{Fe}^{2+}$ ] red complex appear to represent an efficient and economical platform for IgG purification. The possibility of integrating this platform into industrial, continuous production flows is supported by the findings that: (i)filtration can replace centrifugation and lead to highly pure, recovered Abs $(>95 \%)$ with improved overall recovery yields (79-90\%); (ii) process efficiency is not significantly reduced at higher IgG concentrations (e.g. $3-5 \mathrm{mg} / \mathrm{mL}$ ); and (iii)chelator recycling is almost quantitative (95\%). (iv) The process is rapid: on average, only 15-20 minutes are needed to capture the target IgG and recover it in pure form via the filtration protocol. This should translate into significant time saving and hence, higher manufacturing productivity. However, the true practical and economic value of the IgG purification platform for downstream processing of therapeutic grade mAbs will now require additional process upscaling.

\section{Acknowledgments}

We thank the Kimmelman Center for Biomolecular Structure and Assembly, the Benoziyo Endowment Fund for the Advancement of Science ; and the J \& R Center for Scientific Research for their generous support to M.S. M. S. holds the Katzir-Makineni Chair in Chemistry. G. P. thanks Ariel University for its support. We wish to thank Dr. Rami Kriger for his assistance with the analysis of the LC-MS samples. G. P. thank Ariel University for their support.

\section{Materials and methods}

Materials: Ethylenediaminetetraacetic acid (Sigma, E6758), Bovine serum albumin (Sigma, A7906), Protein A HP Spin-Trap (Sigma, 28903132), iron (II) sulfate heptahydrate (Sigma, F7002), sodium chloride (Sigma, S7653), polysorbate 20 (Sigma, 44112), polysorbate 40 (Sigma, P1504), polysorbate 60 (Sigma, P1629), polysorbate 80 (Sigma, P1754), Brij C-10 (Sigma, 388858), Brij O-20 (Sigma, 436240), Brij S-100 (Sigma, 466387), Triton X-100 (Sigma, laboratory grade), Pluronic F-127 (Sigma, P2443) Ex-CELL 610HSF medium (Sigma, 14610C), glycine (Bio-lab 07132391), bathophenanthroline (GFS chemicals, C038446), streptavidin-HRP conjugate (RD system, 321894), TMB solution (eBioscience,:00-4201), PBS buffer 10X (Bio-Lab, 00162323G500), human IgG (Lee-Biosicences, 340-21), mouse IgG (Equitech, SLM66).

\section{Preparation of detergent aggregates}

Detergent aggregates were obtained by mixing equal volumes of medium A and B as follows: medium A was prepared by the addition of $20 \mu \mathrm{L}$ of the hydrophobic chelator bathophenanthroline (20 $\mathrm{mM}$ in methanol) to $180 \mu \mathrm{L}$ of: $0.25 \mathrm{mM} ; 0.215 \mathrm{mM} ; 0.062 \mathrm{mM} ; 0.062 \mathrm{mM} ; 0.1 \mathrm{mM} ; 0.09 \mathrm{mM} ; 0.05 \mathrm{mM} ; 0.1 \mathrm{mM}$ and 0.5 $\mathrm{mg} / \mathrm{mL}$ of Tween-20, Tween-40, Tween-60, Tween-80, Brij C-10, Brij O-20, Brij S-100, Triton X-100 and Pluronic F-127 in DDW, respectively, with vigorous vortexing to a final volume of $200 \mu \mathrm{L}$. An equal volume of medium B , containing $1 \mathrm{mM} \mathrm{FeSO} 4$ in $20 \mathrm{mM} \mathrm{NaCl}$ was then added to medium A with vigorous vortexing.

\section{Purification of hIgG and mouse IgG with detergent aggregates}

Freshly prepared aggregates were resuspended in $100 \mu \mathrm{L}$ of serum-free medium (Ex-CELL 610-HSF) containing: 4\% PEG-6000, the target $\operatorname{IgG}(1 \mathrm{~g} / \mathrm{mL})$ and BSA $(0.25 \mathrm{mg} / \mathrm{mL})$. After 5 minutes of incubation at room temperature, centrifugation $(14 \mathrm{~K}, 2 \mathrm{~min}$.) was applied, the supernatant discarded and pellets were briefly washed with $100 \mu \mathrm{L}$ of cold $20 \mathrm{mM} \mathrm{NaCl}$. An additional centrifugation step followed (14K rpm, 2 min.), the supernatant was removed, and pellets were analyzed by SDS-PAGE.

\section{Extraction of IgG's from detergent aggregates}

Detergent aggregates containing the target IgG were incubated with $100 \mu \mathrm{L}$ of: $50 \mathrm{mM}$ Gly, $30 \mathrm{mM} \mathrm{NaCl}$ at pH 3.8 for 5 minutes at room temperature. Centrifugation followed (13K rpm, $2 \mathrm{~min}$.) and the supernatant was carefully removed for further analysis by pipetation or filtered using PVDF filters with a pore size of $0.1 \mu \mathrm{m}$ (Millipore - Ultrafree @ MC- VV).

\section{Comparison with Protein A spin columns}

The IgG:BSA sample, prepared as described above, was applied to commercial Protein A spin column and 
purification was performed according to manufacturer instructions (General Electric). Eluted IgG's were then compared to those purified using Tween-20, Tween-40, Tween-60, Tween-80, Brij C-10, Brij O-20, Brij S-100, Triton X-100 and Pluronic F-127 aggregates.

\section{Dynamic light scattering (DLS)}

IgG $(0.5-1.0 \mathrm{mg} / \mathrm{mL})$, treated and untreated, was solubilized in $50 \mathrm{mM}$ glycine and $30 \mathrm{mM} \mathrm{NaCl}$ at $\mathrm{pH} 3.8$. Samples were centrifuged at $13 \mathrm{~K} \mathrm{rpm}$ for 20 minutes and the supernatant collected for analysis. The intensityweighted size distributions of human and mouse IgG samples were determined using the auto correlation spectroscopy protocol of the Nanophox instrument (Sympatec GmbH, Germany).

\section{Enzyme-linked immunosorbent assay (ELISA)}

Nunc-Immuno Microwell plates (F96 Maxisorp) were first coated with 2\% BSA (200 $\mu \mathrm{L}$ ), left for overnight incubation at $4{ }^{\circ} \mathrm{C}$ and excess of BSA was removed with three aliquots of PBS $(200 \mu \mathrm{L})$. Purified anti-BSA IgGs, i.e ., IgGs that were captured by either Tween-20, Brij-O20 or Triton X-100 aggregates and extracted with Gly buffer (see the Extraction section of IgG's from detergent aggregates), were then added to the washed wells. Accordingly, $100 \mu \mathrm{L}$ of purified and diluted (1:1000) naked anti-BSA IgG (from rabbit) were added to the BSA coated wells, incubated for 2 hours at room temperature (RT) and unbound anti-BSA IgG's were then removed with PBS $(3 \times 200 \mu \mathrm{L})$. To wells containing the anti-BSA IgG, a diluted $(1: 15,000)$ anti-rabbit IgG-HRP conjugate was introduced into wells containing the naked IgG. In both cases, the system was further incubated for 1 hour at RT and excess HRP conjugates (either streptavidin or IgG) were excluded with PBS $(3 \times 200 \mu \mathrm{L})$. Addition of the HRP substrate (1 X TMB solution) was followed by 10 minutes of incubation at RT and the reaction was stopped upon addition of $2 \mathrm{~N} \mathrm{H}_{2} \mathrm{SO}_{4}(50 \mu \mathrm{L})$. The intensity of the yellow color in the wells was measured at $450 \mathrm{~nm}$ using an ELISA reader (Tecan infinite M200).

\section{Chelator recycyling}

Batho regeneration was achieved via three sequential steps illustrated in Figure 5: Step I: Removal of Tween20. Red Tween-20 aggregates, from which hIgG was extracted, were resuspended in $200 \mu \mathrm{l}$ of $0.25 \mathrm{M} \mathrm{NaCl}$, vortexed for 1 minute, followed by short spin (13K, 2 minutes). The supernatant was discarded and the process was repeated two more times. Step II: Dissociation of the red $\left[(\text { batho })_{3}: \mathrm{Fe}^{2+}\right]$ complex. The resulting red crystals (Figure 6, A) were resuspended in $800 \mu \mathrm{l}$ comprised of $\mathrm{MeOH} \backslash \mathrm{DDW}$ (1:4 ratio) containing 0.25 $\mathrm{M}$ EDTA ( $\mathrm{pH} 7$ ) and heated for 3 minutes at $80^{\circ} \mathrm{C}$. The suspension red color, gradually decreased and the solution became colorless and transparent. Step III: Crystal growth. The colorless transparent medium was left to cool to room temperature (20-30 minutes) and further to $4{ }^{\circ} \mathrm{C}(20-30$ minutes). Colorless plate crystals with a range of sizes $(20-150 \mu \mathrm{m})$ were observed (Figure 6, B).

\section{Liquid chromatography mass spectrometry (LCMS)}

Red or colorless crystals were thoroughly washed with DDW and dissolved in $\mathrm{MeOH}$. Liquid chromatography mass spectrometry (LC/MS) was performed with the Agilent quadruple LC/MS 6120 series equipped with Reprosil saphir 100, C18, $50 \times 2 \mathrm{~mm}$, HPLC column. The eluent solvents were $100 \% \mathrm{CH}_{3} \mathrm{CN}$. The UV detection was at $254 \mathrm{~nm}$. The column temperature was kept at $50{ }^{\circ} \mathrm{C}$. The flow rate was $0.4 \mathrm{ml} / \mathrm{min}$. The MS fragmentor was tuned on $30 \mathrm{~V}$ or $70 \mathrm{~V}$ in positive or negative mode.

\section{UV spectroscopy}

Absorption measurements of the red $\left[(\text { batho })_{3}: \mathrm{Fe}^{2+}\right]$ complex $(\lambda \max =530 \mathrm{~nm})$, dissolved in $\mathrm{MeOH}$, were performed using the HP $8453 \mathrm{UV}$-Vis spectrophotometer.

\section{Densitometry}

Bands present in Coomassie stained gels were quantified using the EZQuant program. http://www.ezquant.com/en/

\section{Light microscopy}


Images were obtained using an Olympus CX-40 light microscope equipped with an Olympus U-TV1X-2 digital camera.

\section{References}

1. Alkan, S. S., Monoclonal antibodies: the story of a discovery that revolutionized science and medicine. Nature reviews. Immunology 2004, 4 (2), 153-6.

2. Weiner, G. J., Building better monoclonal antibody-based therapeutics. Nature reviews. Cancer $\mathbf{2 0 1 5}$, 15 (6), 361-70.

3. Liu, J. K., The history of monoclonal antibody development - Progress, remaining challenges and future innovations. Annals of medicine and surgery (2012)2014, 3 (4), 113-6.

4. Mullard, A., Maturing antibody-drug conjugate pipeline hits 30. Nature reviews. Drug discovery2013, $12(5), 329-32$.

5. Sievers, E. L.; Senter, P. D., Antibody-drug conjugates in cancer therapy. Annual review of medicine 2013, $64,15-29$.

6. Strohl, W. R., Current progress in innovative engineered antibodies. Protein \& cell 2018,9 (1), 86-120.

7. Kaplon, H.; Reichert, J. M., Antibodies to watch in 2019. mAbs 2019, 11 (2), 219-238.

8. Hober, S.; Nord, K.; Linhult, M., Protein A chromatography for antibody purification. Journal of Chromatography B 2007, 848 (1), 40-47.

9. DeLano, W. L.; Ultsch, M. H.; de Vos, A. M.; Wells, J. A., Convergent solutions to binding at a proteinprotein interface. Science (New York, N.Y.)2000, 287 (5456), 1279-83.

10. Page, M.; Thorpe, R., Purification of IgG Using Protein A or Protein G. In The Protein Protocols Handbook, Walker, J. M., Ed. Humana Press: Totowa, NJ, 2002; pp 993-994.

11. Azevedo, A. M.; Gomes, A. G.; Rosa, P. A. J.; Ferreira, I. F.; Pisco, A. M. M. O.; Aires-Barros, M. R., Partitioning of human antibodies in polyethylene glycol-sodium citrate aqueous two-phase systems. Separation and Purification Technology 2009, 65 (1), 14-21.

12. Low, D.; O'Leary, R.; Pujar, N. S., Future of antibody purification. Journal of chromatography. B, Analytical technologies in the biomedical and life sciences2007, 848 (1), 48-63.

13. Ecker, D. M.; Jones, S. D.; Levine, H. L., The therapeutic monoclonal antibody market. mAbs 2015, 7 (1), 9-14.

14. Gagnon, P., Technology trends in antibody purification. J Chromatogr A 2012, 1221, 57-70.

15. Wurm, F. M., Production of recombinant protein therapeutics in cultivated mammalian cells. Nat Biotechnol 2004, 22 (11), 1393-8.

16. Linhult, M.; Gulich, S.; Graslund, T.; Simon, A.; Karlsson, M.; Sjoberg, A.; Nord, K.; Hober, S., Improving the tolerance of a protein a analogue to repeated alkaline exposures using a bypass mutagenesis approach. Proteins 2004, 55 (2), 407-16.

17. McDonald, P.; Victa, C.; Carter-Franklin, J. N.; Fahrner, R., Selective antibody precipitation using polyelectrolytes: a novel approach to the purification of monoclonal antibodies. Biotechnology and bioengineering2009, 102 (4), 1141-51.

18. Azevedo, A. M.; Rosa, P. A.; Ferreira, I. F.; Aires-Barros, M. R., Chromatography-free recovery of biopharmaceuticals through aqueous two-phase processing. Trends in biotechnology 2009, 27 (4), 240-7.

19. Mao, L. N.; Rogers, J. K.; Westoby, M.; Conley, L.; Pieracci, J., Downstream antibody purification using aqueous two-phase extraction. Biotechnology progress2010, 26 (6), 1662-70. 
20. van Reis, R.; Zydney, A., Bioprocess membrane technology. Journal of Membrane Science 2007, 297 (1-2), 16-50.

21. Dhandapani, G.; Howard, A.; Truong, T. V.; Baiju, T. V.; Kesselman, E.; Friedman, N.; Wachtel, E.; Sheves, M.; Danino, D.; Namboothiri, I. N. N.; Patchornik, G., A general platform for antibody purification utilizing engineered-micelles.mAbs 2019, 11 (3), 583-592.

22. Dhandapani, G.; Nair, D. K.; Kale, R. R.; Wachtel, E.; Namboothiri, I. N. N.; Patchornik, G., Role of amphiphilic [metal:chelator] complexes in a non-chromatographic antibody purification platform. Journal of chromatography. B, Analytical technologies in the biomedical and life sciences 2019, 1133 , 121830.

23. Manzke, O.; Tesch, H.; Diehl, V.; Bohlen, H., Single-step purification of bispecific monoclonal antibodies for immunotherapeutic use by hydrophobic interaction chromatography. Journal of Immunological Methods 1997, 208 (1), 65-73.

24. Guse, A. H.; Milton, A. D.; Schulze-Koops, H.; Muller, B.; Roth, E.; Simmer, B.; Wachter, H.; Weiss, E.; Emmrich, F., Purification and analytical characterization of an anti-CD4 monoclonal antibody for human therapy. J Chromatogr A1994, 661 (1-2), 13-23.

25. Follman, D. K.; Fahrner, R. L., Factorial screening of antibody purification processes using three chromatography steps without protein A. J Chromatogr A2004, 1024 (1-2), 79-85.

26. Ghosh, R.; Wang, L., Purification of humanized monoclonal antibody by hydrophobic interaction membrane chromatography. Journal of Chromatography A 2006,1107 (1), 104-109.

27. Patchornik, G.; Namboothiri, I. N. N.; Nair, D. K.; Wachtel, E.; Persky, R., Tethered non-ionic micelles: a matrix for enhanced solubilization of lipophilic compounds.Soft matter 2012, 8 (32), 8456-8463.

28. Patchornik, G.; Wachtel, E.; Kesselman, E.; Danino, D., Cryo-TEM structural analysis of conjugated nonionic engineered-micelles. Soft matter 2014,10 (27), 4922-8.

29. Dutta, S.; Nair, D. K.; Namboothiri, I. N.; Wachtel, E.; Friedman, N.; Sheves, M.; Patchornik, G., Engineered-membranes and engineered-micelles as efficient tools for purification of halorhodopsin and bacteriorhodopsin. The Analyst2015, 140 (1), 204-12.

30. Martell, A. E.; Smith, R. M.,Critical stability constants . Plenum Press: New York, 1974.

31. Roberts, C. J., Therapeutic protein aggregation: mechanisms, design, and control. Trends in biotechnology 2014, 32 (7), 372-80.

32. Dhandapani, G.; Howard, A.; Truong, T. V.; Baiju, T. V.; Kesselman, E.; Friedman, N.; Wachtel, E.; Sheves, M.; Danino, D.; Namboothiri, I. N. N.; Patchornik, G., A general platform for antibody purification utilizing engineered-micelles. $m A b s 2019$.

33. Liu, H.; May, K., Disulfide bond structures of IgG molecules. mAbs 2012, 4 (1), 17-23.

34. Kunert, R.; Reinhart, D., Advances in recombinant antibody manufacturing. Appl Microbiol Biotechnol 2016, 100 (8), 3451-3461.

35. Irving, H.; Mellor, D. H., 1002. The stability of metal complexes of 1,10-phenanthroline and its analogues. Part I. 1,10-Phenanthroline and 2,2'-bipyridyl. Journal of the Chemical Society (Resumed) 1962, (0), 52225237.

36. Derman, D. P.; Green, A.; Bothwell, T. H.; Graham, B.; McNamara, L.; MacPhail, A. P.; Baynes, R. D., A systematic evaluation of bathophenanthroline, ferrozine and ferene in an ICSH-based method for the measurement of serum iron. Annals of clinical biochemistry 1989, 26 ( Pt 2) , 144-7.

37. Mabrouk, P. A., Green Chemistry and Catalysis By Roger A. Sheldon, Isabel W. C. E. Arends, and Ulf Hanefeld (Delft University of Technology, The Netherlands). Wiley-VCH Verlag GmbH \& Co. KGaA: 
Weinheim. 2007. xiv +434 pp. \$190. ISBN 978-3-527-30715-9. Journal of the American Chemical Society 2008, 130 (8), 2704-2704.

Figure legends

Figure 1: A. Illustration of $\mathbf{A b}(\mathrm{IgG})$ purification using conjugated micellar aggregates. Target IgG + BSA are captured by micellar aggregates comprised of a non-ionic detergent, amphiphilic chelator (e.g. bathophenanthroline, (batho)) and $\mathrm{Fe}^{2+}$ ions. Pure IgG is obtained by extracting IgG's bound to the micellar aggregates under conditions that do not co-extract BSA or dissociate the detergent aggregates. B. Chemical structure of the non-ionic detergents. The sum of the values represented by the letters: W, X, Y, $\mathrm{Z}$ in the Tween-family is 20. The number of carbons comprising the hydrophobic anchor of: Tween-20, 40, 60 and 80, are indicated, respectively.

Figure 2: A. SDS-PAGE analysis of captured IgG's in pellets containing non-ionic detergents. A . Pellet composition: Lane 1: Control: total hIgG and BSA; Lanes 2-5: Pellet composition after hIgG capture in the presence of the Tween representatives; Lanes 6-8: as in lanes 2-5 but in the presence of Brij representatives; Lane 9 and 10 as in lanes 2-5 but in the presence of Triton X-100 and Pluronic F-127, respectively. B . As inA, but with mouse IgG. BSA , H , L,A denote bovine serum albumin, the reduced $\mathbf{h}$ eavy chain, the reduced $\mathbf{l}$ ight chain and the detergenta ggregates, respectively. C-D . Supernatant composition after extracting IgG's from indicated [detergent:[(batho $)_{3}: \mathrm{Fe}^{2+}$ ] pellets with $50 \mathrm{mM}$ glycine at pH 3.8 as described in Experimental. Indicated overall process yields (i.e. IgG capture and extraction) are shown and represent an average of 3 independent experiments. Gels are Coomassie stained.

Figure 3: Dynamic light scattering (DLS) of detergent aggregates. Hydrodynamic $\mathbf{p}$ article size of detergent aggregates containing the non-ionic detergents and the $\left[(\text { batho })_{3}: \mathrm{Fe}^{2+}\right]$-complex. The data represent an average of at least 5 independent measurements performed on different days.

Figure 4: Purification of IgG's via filtration.A. Characterization of detergent aggregates for purifying hIgG under conditions described in the Experimental section, where centrifugation is replaced by filtration. BSA , H , L denote bovine serum albumin, the reduced $\mathbf{h}$ eavy chain, the reduced $\mathbf{l}$ ight chain, respectively. The asterisks represent overall yields based on 4-6 independent experiments. Gels are Coomassie stained. B. Dynamic light scattering (DLS) analysis of hIgG purified via filtration with detergents as indicated. Control - hIgG particle size $(10.95 \mathrm{~nm})$, not subject to detergent aggregate purification. C. ELISA analysis. Anti-BSA IgG was captured and extracted from indicated detergent aggregates and tested for its ability to recognize BSA via an ELISA assay described in the Experimental. Indicated percent values represent IgG recovery yields relative to equal amounts of IgG not exposed to detergent aggregates (control). Filters used were made of PVDF with a pore size of $0.1 \mu \mathrm{m}$ (Millipore - Ultrafree $(\mathrm{R}) \mathrm{MC}-\mathrm{VV}$ ).

Figure 5: Overall recovery yields with detergent aggregates as a function of hIgG concentration. The asterisk represents a purification protocol identical to the one described in the Experimental section except for the following changes that were introduced during aggregate formation: (i) $\mathrm{FeSO}_{4}$ was replaced by $\mathrm{FeCl}_{2}$ at the same concentration. (ii) $10 \mathrm{mM} \mathrm{NaCl}$ was increased to $200 \mathrm{mM} \mathrm{NaCl}$.

Figure 6: Chelator recycling. Batho recycling via recrystallization. Yield represents an average of 5 independent experiments.

Figure 7: Light microscopy images of crystals obtained at steps indicated in Figure 6.

\section{Figures}



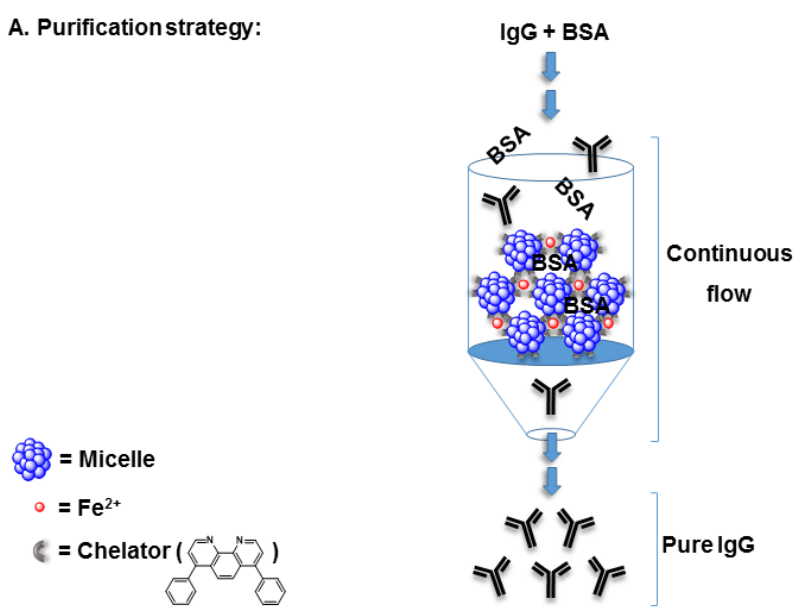

B.

Tween-family $(20,40,60,80)$

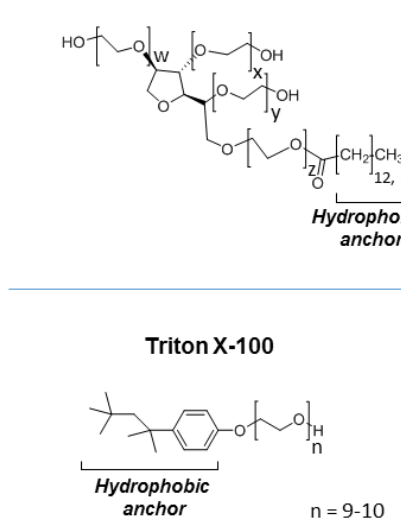

Brij-family $(10,20,100)$

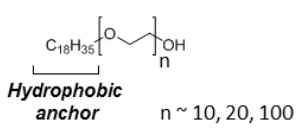

Pluronic F-127

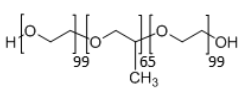

Tri block co-polymer

Figure 1: A. Illustration of $\mathrm{Ab}(\mathrm{IgG})$ purification using conjugated micellar aggregates. Target IgG + BSA are captured by micellar aggregates comprised of a non-ionic detergent, amphiphilic chelator (e.g. bathophenanthroline, (batho)) and $\mathrm{Fe}^{2+}$ ions. Pure IgG is obtained by extracting IgG's bound to the micellar aggregates under conditions that do not co-extract BSA or dissociate the detergent aggregates. B. Chemical structure of the non-ionic detergents. The sum of the values represented by the letters: W, X, Y, $\mathrm{Z}$ in the Tween-family is 20. The number of carbons comprising the hydrophobic anchor of: Tween-20, 40, 60 and 80, are indicated, respectively. 
A. hlgG capture (pellet composition)

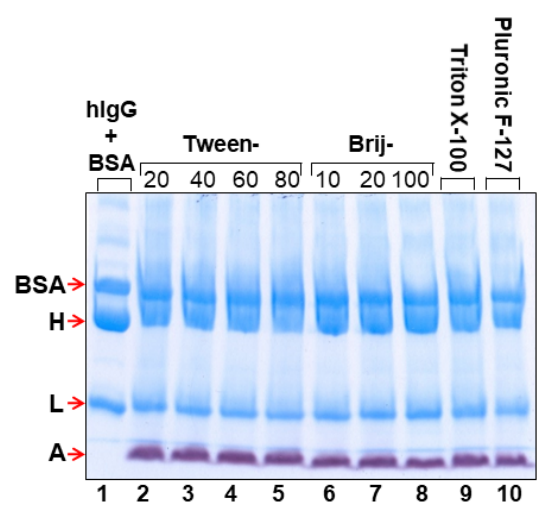

C. RecoveredhlgG (supernatant)

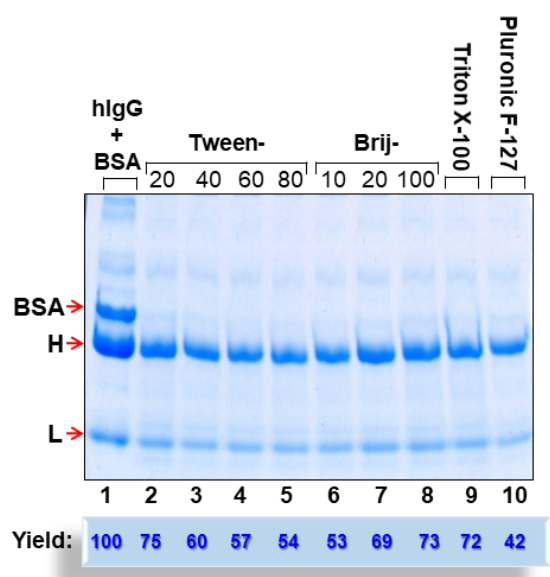

B. Mouse IgG capture (pellet composition)

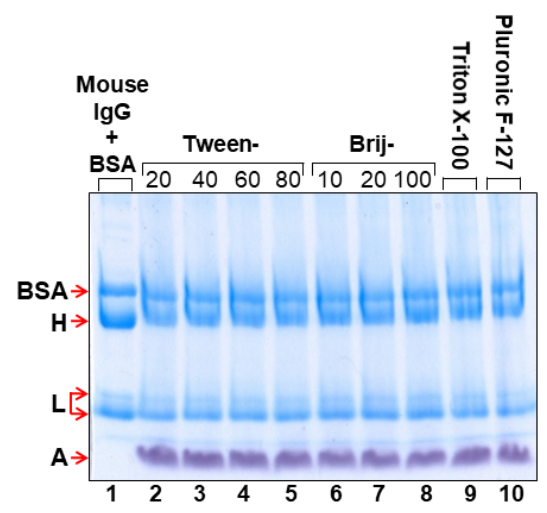

D. Recoveredmouse IgG (supernatant)

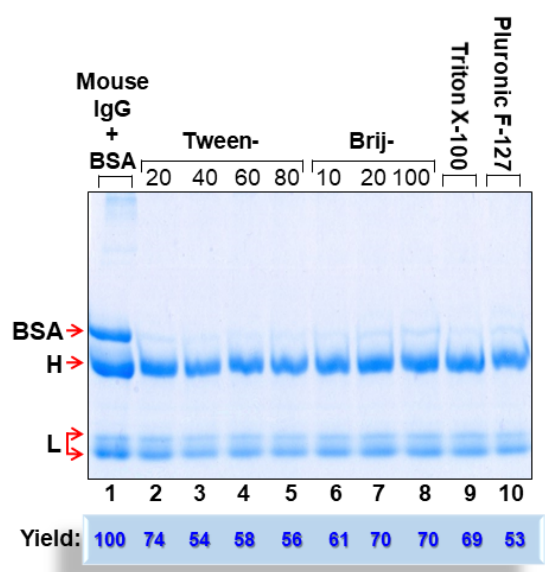

Figure 2: A. SDS-PAGE analysis of captured IgG's in pellets containing non-ionic detergents. A . Pellet composition: Lane 1: Control: total hIgG and BSA; Lanes 2-5: Pellet composition after hIgG capture in the presence of the Tween representatives; Lanes 6-8: as in lanes 2-5 but in the presence of Brij representatives; Lane 9 and 10 as in lanes 2-5 but in the presence of Triton X-100 and Pluronic F-127, respectively. B . As in A, but with mouse IgG. BSA , H , L,A denote bovine serum albumin, the reduced $\mathbf{h}$ eavy chain, the reduced $\mathbf{l}$ ight chain and the detergenta ggregates, respectively. C-D . Supernatant composition after extracting IgG's from indicated [detergent:[(batho $)_{3}: \mathrm{Fe}^{2+}$ ] pellets with $50 \mathrm{mM}$ glycine at $\mathrm{pH} 3.8$ as described in Experimental. Indicated overall process yields (i.e. IgG capture and extraction) are shown and represent an average of 3 independent experiments. Gels are Coomassie stained. 
A. Tween-family

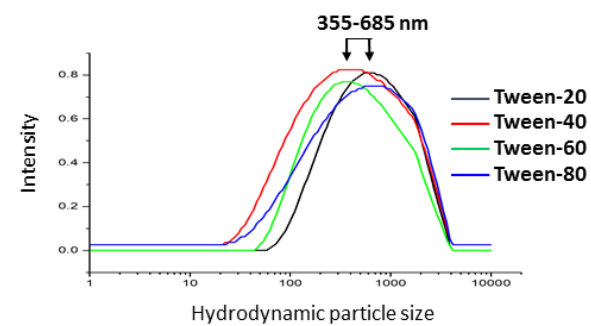

C. Triton X-100

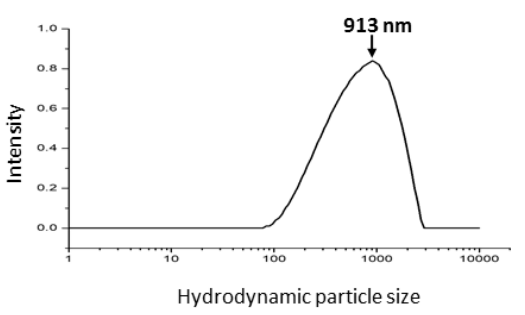

B. Brij-family

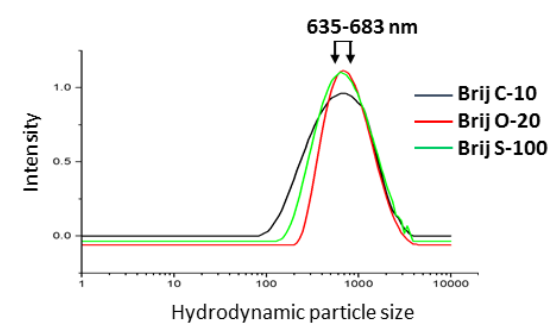

D. Pluronic F-127

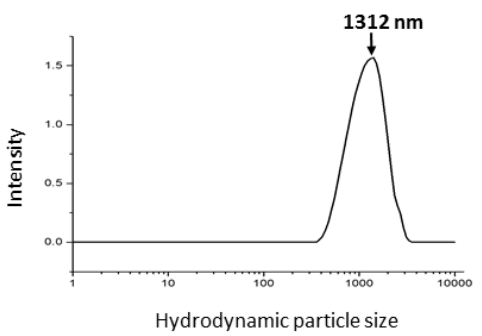

Figure 3: Dynamic light scattering (DLS) of detergent aggregates. Hydrodynamic $p$ article size of detergent aggregates containing the non-ionic detergents and the $\left[(\text { batho })_{3}: \mathrm{Fe}^{2+}\right]$-complex. The data represent an average of at least 5 independent measurements performed on different days.

A. Filtration (hlgG)
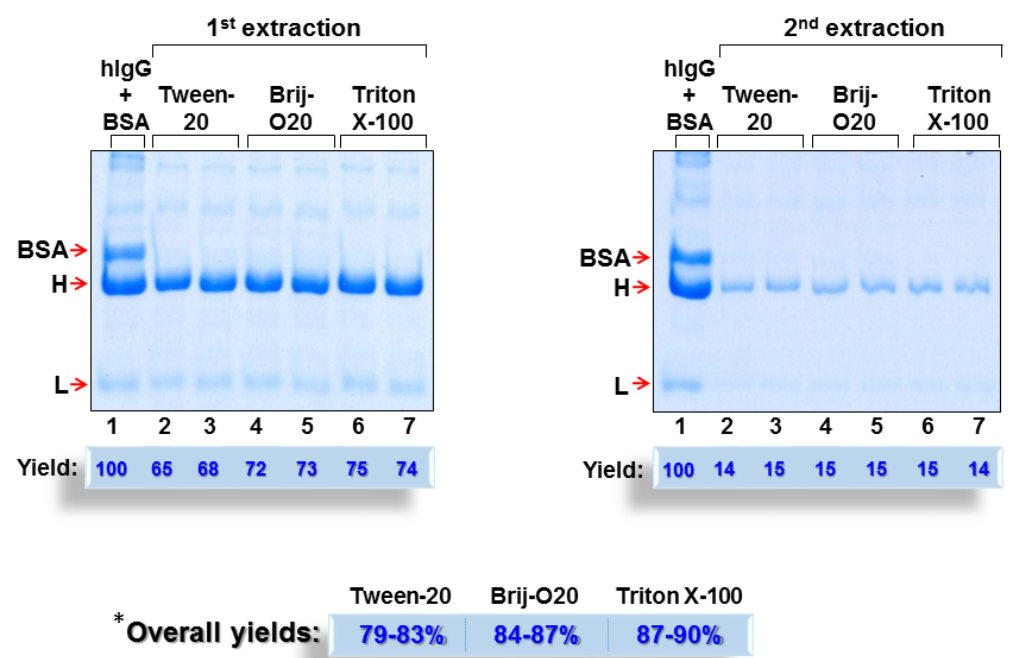


\section{B. Filtration: DLS (hlgG)}

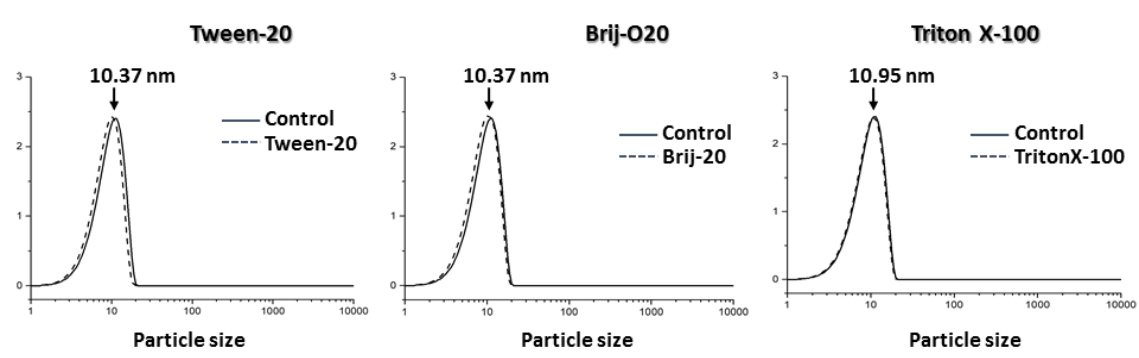

\section{Filtration: ELISA (anti-BSA IgG)}

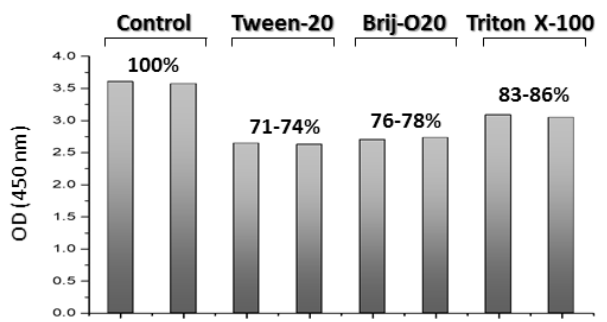

Figure 4: Purification of IgG's via filtration.A. Characterization of detergent aggregates for purifying hIgG under conditions described in the Experimental section, where centrifugation is replaced by filtration. BSA , H , L denote bovine serum albumin, the reduced $\mathbf{h}$ eavy chain, the reduced $\mathbf{l}$ ight chain, respectively. The asterisks represent overall yields based on 4-6 independent experiments. Gels are Coomassie stained. B. Dynamic light scattering (DLS) analysis of hIgG purified via filtration with detergents as indicated. Control - hIgG particle size $(10.95 \mathrm{~nm})$, not subject to detergent aggregate purification. C. ELISA analysis. Anti-BSA IgG was captured and extracted from indicated detergent aggregates and tested for its ability to recognize BSA via an ELISA assay described in the Experimental. Indicated percent values represent IgG recovery yields relative to equal amounts of IgG not exposed to detergent aggregates (control). Filters used were made of PVDF with a pore size of $0.1 \mu \mathrm{m}$ (Millipore - Ultrafree $\AA$ MC- VV).

\begin{tabular}{|c|c|c|c|}
\hline & Tween-20 & Brij-O20 & Triton X-100 \\
\hline $1 \mathrm{mg} / \mathrm{ml} \mathrm{hlgG}$ & $79-83 \%$ & $84-87 \%$ & $87-90 \%$ \\
\hline $3 \mathrm{mglml} \mathrm{hlgG} \mathrm{*}$ & $74-78 \%$ & $80-85 \%$ & $82-87 \%$ \\
\hline $5 \mathrm{mg} / \mathrm{ml} \mathrm{hlgG}$ * & $70-76 \%$ & $78-83 \%$ & $76-79 \%$ \\
\hline
\end{tabular}

Figure 5: Overall recovery yields with detergent aggregates as a function of hIgG concentration. The asterisk represents a purification protocol identical to the one described in the Experimental section except for the following changes that were introduced during aggregate formation: (i) $\mathrm{FeSO}_{4}$ was replaced by $\mathrm{FeCl}_{2}$ at the same concentration. (ii) $10 \mathrm{mM} \mathrm{NaCl}$ was increased to $200 \mathrm{mM} \mathrm{NaCl}$. 


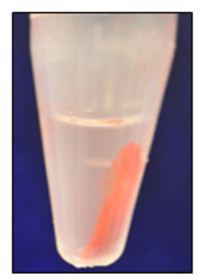

Pellet after

Ab extraction

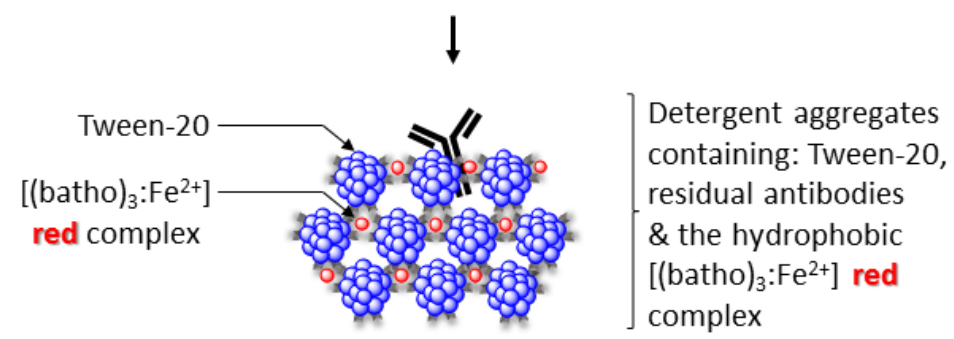

Step I:

Removal of most Tween-20

\& IgG that wasn't extracted.
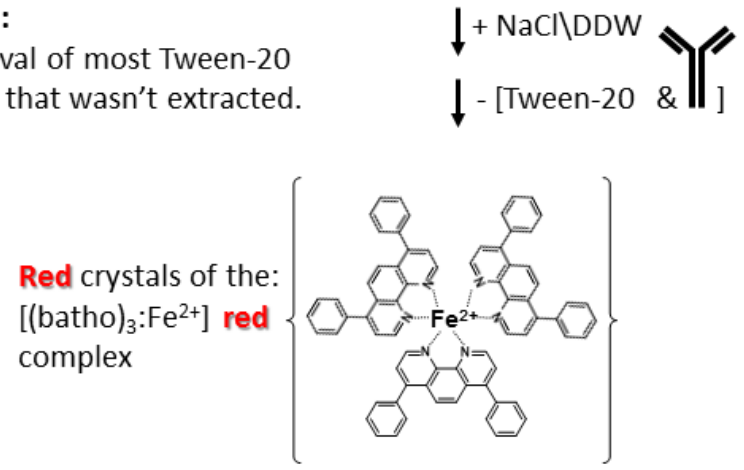

Step II:

Dissociation of the $\left[(\text { batho })_{3}: \mathrm{Fe}^{2+}\right]$ red $\downarrow$ complex with a competing chelator: EDTA. Loss of red color.

+EDTA (excess) $\backslash D D W, \mathrm{CH}_{3} \mathrm{OH}$ $95^{\circ} \mathrm{C}, 2-3 \mathrm{~min}$.

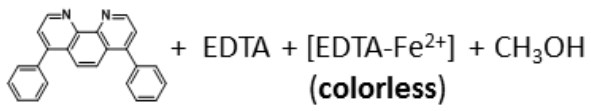

Step III:

Evaporation of water \& $\mathrm{CH}_{3} \mathrm{OH}$. $\downarrow$

Step IV:

Removal of EDTA

$\downarrow$

+ DDW (-EDTA \& [EDTA-Fe $\left.\left.{ }^{2+}\right]\right)$

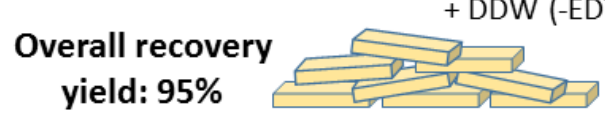

Crystals of recycled chelator

Figure 6: Chelator recycling. Batho recyclingvia recrystallization. Yield represents an average of 5 independent experiments. 
A. Crystals observed after: "Step I"

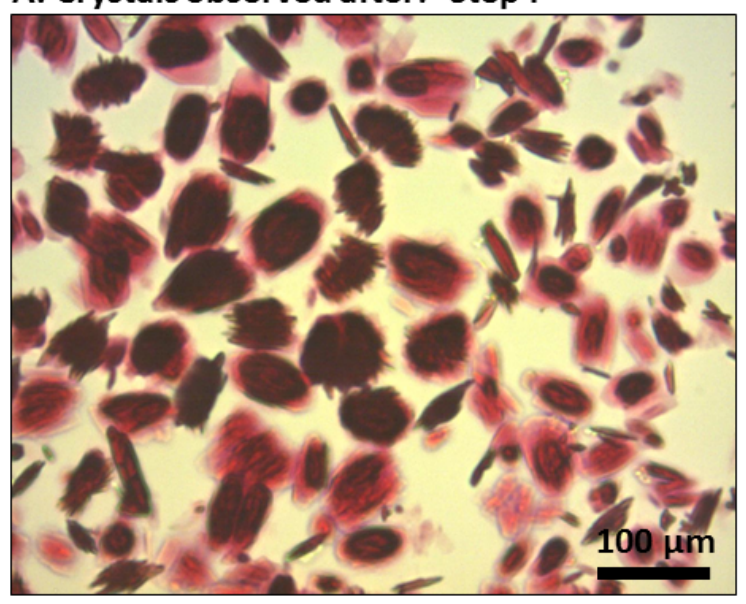

B. Crystals observed after: "Step IV"

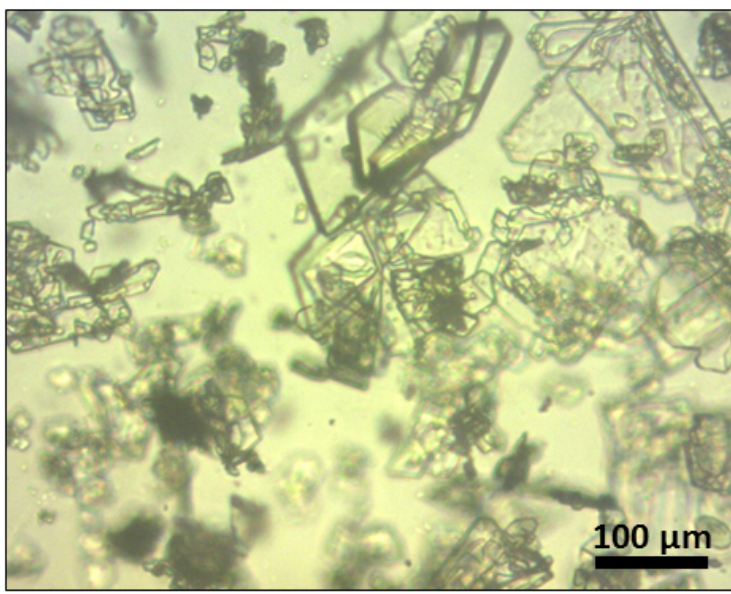

C. Batho crystals prior to their use (control)

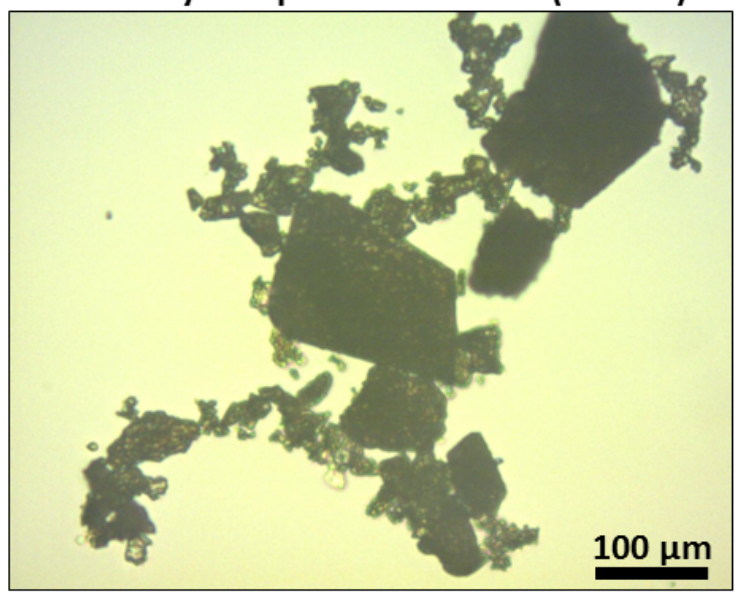

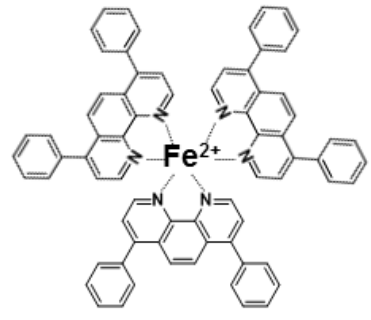

[(batho $\left.)_{3}: \mathrm{Fe}^{2+}\right]$ red complex?

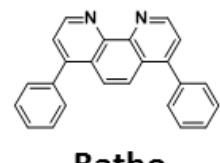

Batho

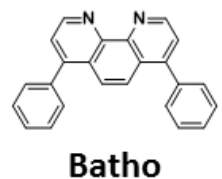

Figure 7: Light microscopy images of crystals obtained at steps indicated in Figure 6. 


\section{Hosted file}

Image . docx available at https ://authorea.com/users/145740/articles/428148-nonionic-detergentmicelle-aggregates-an-economical-alternative-to-protein-a-chromatography 